\title{
Cultura, CAMbio y APRENDizaje EN LAS ORGANIZACIONES BASADAS EN PROYECTOS
}

\author{
Culture, CHANGE AND LEARNING \\ IN PROJECT-BASED ORGANIZATIONS
}

\section{PAUL IDROBO DÁVALOS}

Pontificia Universidad Católica del Ecuador

Correo electrónico: pmidrobo@puce.edu.ec

\section{FABIÁN CUEVA BRITO}

Pontificia Universidad Católica del Ecuador

Fecha de RECEPCIÓN: 05/05/2016

Correo electrónico: efcuevab@puce.edu.ec

FECHA DE ACEPTACIÓN: 05/06/2016

\section{RESUMEN}

Los proyectos son el medio estratégico por el cual las organizaciones implementan sus iniciativas de mejora; sin embargo, no todas son capaces de gestionarlos de manera tal que se asegure su desempeño. Este artículo analiza la incidencia de la cultura de las organizaciones, su adaptabilidad al cambio y su capacidad de aprendizaje, como elementos claves para que estas puedan basar su gestión en proyectos, a la par que propone buenas prácticas que permitan incrementar sus posibilidades de éxito en este desafío.

Palabras clave: Organizaciones basadas en proyectos (OBP), Gestión de proyectos.

\section{Abstract}

Projects are the strategic means by which organizations implement their improvement initiatives, but not all are able to manage them in a way that ensures their performance. This article analyzes the impact of organizational culture, its adaptability to change and its learning capacity, as key elements for them to base their management on projects, while proposing good practices that increase their chances of success in this challenge.

Key words: Project-based organizations (PBOs), project management.

ClasificaCión JEL: M14 Cultura corporativa, M10 Generalidades 


\section{Introducción}

Un proyecto es un proceso único consistente en un conjunto de actividades coordinadas y controladas con fechas de inicio y de finalización, llevadas a cabo para lograr un objetivo conforme con requisitos específicos, incluyendo las limitaciones de tiempo, costo y recursos (ISO 10006, 2003). Los proyectos se utilizan comúnmente como una herramienta estratégica para cumplir con los objetivos de las organizaciones. Los proyectos suelen crearse como respuesta a una o más de las siguientes consideraciones estratégicas: demanda del mercado, oportunidad estratégica/necesidad comercial, solicitud de un cliente, adelantos tecnológicos y requisitos legales (PMI, 2013).

La gestión del proyecto involucra planeación, delegación, monitoreo y control de todos los aspectos del proyecto y la motivación de los involucrados, para alcanzar los objetivos del proyecto, dentro de los objetivos de desempeño esperados para: tiempo, costo, calidad, alcance, beneficios y riesgos (OGC, 2009). Para Domingo Ajenjo (2000), el concepto de «dirección de proyectos» es más ambicioso que el de gestión, puesto que esta implica actividades de mayor responsabilidad, que requieren de mayor experiencia y capacitación, tales como: la selección y motivación de equipos de trabajo, el liderazgo, la toma de decisiones estratégicas y otras actividades de índole más humana y cultural que la mera gestión de recursos.

Lindkvist (2004) sostiene que los proyectos no están fuera de un contexto, sino que siempre forman parte de las rutinas y redes de relaciones existentes dentro de una organización. Sin embargo, los proyectos tienen una naturaleza temporal, compleja e independiente. Todo esto, junto a las constantes condiciones cambiantes para los proyectos y los cambios que se producen en las propias organizaciones, provocan modos de organización y prácticas de gestión con técnicas que no se alinean fácilmente a los procesos que regularmente siguen las organizaciones.

Para Hobday (2000) existen distintos tipos de organizaciones basadas en proyectos, encontrándo- se extremos entre algunas que tienen una estructura pura de proyectos y otras que son de corte funcional, pasando por otras que adoptan una estructura matricial.

Gerdin, Björkander, Henriksson \& Nilsby (2010) afirman que las organizaciones basadas en proyectos tienen características únicas, las cuales conllevan a cierto tipo especial de problemas organizacionales y de gestión. Este tipo de organizaciones tienen un alto nivel de descentralización y de equipos autónomos, lo que desemboca en una desconexión entre los proyectos, lo cual hace que se diferencien mucho de las organizaciones tradicionales de corte burocrático.

Como puede apreciarse, las organizaciones basadas en proyectos están inmersas dentro de un contexto muy particular, lo cual hace muy interesante analizar cómo su cultura, los cambios que en ellas se producen y la forma en que estas aprenden, inciden en su gestión. Para poder comprender mejor la dinámica de estas organizaciones se revisarán algunos referentes teóricos ligados a los aspectos de: cultura, cambio y aprendizaje, aplicados a este tipo de organizaciones, con la finalidad de desprender de estos varias conclusiones de orden práctico para su gestión.

\section{Cultura basada en proyectos}

Schein (2009) plantea que la cultura es un patrón de presunciones tácitas compartidas, que fueron aprendidas por un grupo para resolver sus problemas de adaptación externa e integración interna, que han funcionado suficientemente bien para ser considerados válidos $y$, por lo tanto, deben ser enseñados a los nuevos miembros como la manera correcta de percibir, pensar y sentir en relación con esos problemas.

Schneider (1994) asegura que es posible identificar cuatro tipos distintos de culturas esenciales sobre la base de dos aspectos: el contenido y el proceso. Con respecto al contenido, este puede ser algo que está presente en la actualidad o puede ser una posibilidad. Los procesos, por su parte, 
pueden ser personales o interpersonales. Ajmal \& Koskinen (2009) proponen un esquema de dos ejes que incluyen estos aspectos (Figura 1).

La cultura de control se relaciona con la certeza, la predictibilidad, la seguridad, la exactitud y la dependencia. La cultura de la competencia tiene que ver con el logro, con ganarse una distinción por ser el mejor o el que tiene la más alta calidad. La cultura de la colaboración se relaciona con la afiliación y sinergia dentro de una unidad y conexiones cercanas; busca lo tangible, práctico y útil. Finalmente, la cultura de la cultivación tiene que ver con el significado, su autoactualización, con ideas potenciales, creencias, aspiraciones, inspiración y opciones creativas.

Figura 1. Las CUlturas esenciales

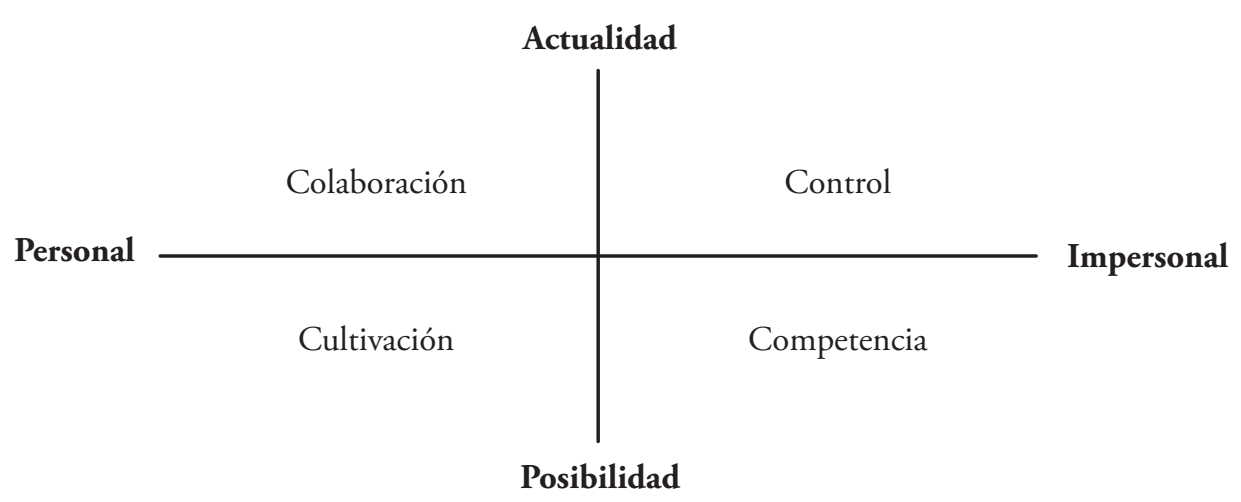

Fuente: Ajmal \& Koskinen (2009)

Es difícil situar a las organizaciones en un tipo específico de los patrones culturales descritos anteriormente, ya que estas al ser dinámicas, generalmente están conformadas por una mezcla de estos. Sin embargo, para Ajmal \& Koskinen (2009), las organizaciones basadas en proyectos deben tener una visión y valores compartidos para dar enfoque y energía a la creación de conocimiento. Además de tener una visión compartida, también es importante que la gente se esfuerce por algo que le importe profundamente en su interior, lo cual propicia la creación del conocimiento generativo.

Para Rosenbloom \& Markus (2010) los directores de proyecto astutos que se embarcan en proyectos de alto impacto, se toman su tiempo para conocer la cultura organizacional y toman pasos proactivos para evaluarla desde cuatro áreas: los valores fundamentales, las recompensas y reconocimientos, la forma cómo se afronta la adversidad y la toma de decisiones.
Lindkvist (2004) considera que una cultura organizacional en donde la alta dirección establece reglas genéricas que sirven para homogenizar las actividades y restablecer el orden no es el ambiente adecuado para las organizaciones basadas en proyectos. Si bien es cierto que es necesario establecer algunas reglas de juego desde el alto mando para mantener un orden mínimo, existen otras reglas que nacen del corazón de la organización y que aseguran un orden que proviene de sus partes, no solo de la cabeza. Se trata de una cultura en donde se privilegia la imaginación individual, la reflexión, la adaptación flexible y la responsabilidad, en oposición a identidades y rutinas prespecificadas.

Según Dinsmore (1997) los cambios que han afectado a las empresas en las últimas décadas han hecho que estas caminen, consciente o inconscientemente, hacia una cultura corporativa de gestión de proyectos, que implica hacer las cosas más rápido, más baratas y de mejor calidad. Para 
este autor se está instaurando en las empresas una cultura de gestión total de proyectos, como en su momento lo hiciera la cultura de gestión de la calidad total. Esta gestión de las organizaciones por proyectos es una filosofía que se basa en el principio que los objetivos de la empresa son alcanzables a través de una red de proyectos simultáneos, lo cual requiere de estrategias corporativas, mejoras operativas y transformación organizacional, así como el desarrollo de los proyectos de corte tradicional. Esto implica que las empresas poco a poco ya no se percibirán a sí mismas como un conjunto de funciones jerárquicas, sino como un conjunto de emprendimientos de rápido seguimiento, constituidos en un portafolio de proyectos en constante cambio y renovación.

Happy \& Meyer (2001) proponen las siguientes prácticas para implementar una cultura efectiva de gestión de proyectos:

- Conocer en primer lugar la estrategia organizacional: misión, visión y objetivos.

- Hacer la implementación por etapas, no todo a la vez.

- Pensar primero en la gente y en los procesos, dejar en segundo lugar el uso de las herramientas.

- Incluir a todos los niveles de la organización.

- Dar a la implementación misma un tratamiento de proyecto.

- Entender que la implementación de la cultura es un proceso de mejora continua.

- Comunicar los éxitos a todos los niveles.

- Ser culturalmente consistente, trabajar hacia soluciones integrales.

La influencia de la cultura en el desarrollo de la estrategia de gestión de proyectos se manifiesta, para Haimes (2001), en al menos seis formas distintas, que son:

- La cultura del equipo del proyecto influye en la forma que se percibe su entorno, tales como los grupos de interés y su papel en el proyecto.

- Los valores y las suposiciones de los equipos del proyecto actúan como filtros, centrando la atención en áreas particulares y lejos de los demás, sesgando así las fortalezas, debilidades, oportunidades y amenazas en las que el equipo se concentra.
- Los miembros del equipo del proyecto interpretan la información obtenida del entorno de una manera particular que se adapte a su propia cultura.

- Se debe decidir cómo responder a los datos interpretados, lo cual puede implicar cuestiones morales y éticas.

- La respuesta adoptada es probable que sea un resultado de un éxito anterior. Asimismo, otras respuestas tal vez se ignoren porque han fracasado en el pasado.

- El equipo de proyecto y su cultura pueden estar de acuerdo en una situación, pero las diversas subculturas ofrecerán diferentes medios de resolverlo.

Schacht (1997) propone las siguientes reglas para que una organización adopte una cultura basada en proyectos de manera exitosa:

- Debe convertirse en una organización que aprende y adopta nuevas ideas, conceptos, y técnicas, y los pone a disposición de todos.

- Establece procesos y medios de comunicación claros, hace que la gente los use y no acepta la comunicación fuera de esos canales.

- Graba y alaba sus logros y a sus héroes, utiliza los éxitos del pasado como modelos para los esfuerzos actuales y futuros.

- Establece una estructura central flexible y se asegura que esta proporciona un núcleo crítico para todos los esfuerzos de implementación.

- Acepta el riesgo y procede juiciosamente; se esfuerza por extender la cultura en toda la organización.

- Se asegura que exista una comprensión común de lo que la cultura está destinada a ser, y lo que no lo es.

- Evalúa y demuestra el valor económico de la cultura.

- Involucra a todos.

\section{El rol de los proyectos en la gestión del cambio}

La gestión del cambio, para Moran \& Brightman (2001), es un proceso de continua renovación de dirección de la organización, su estructura y sus 
capacidades, para servir a las siempre cambiantes necesidades de los clientes externos e internos. Levasseur (2010) afirma que el uso activo de los modelos, metodologías y procesos de la gestión del cambio, aumentan las posibilidades de éxito en los proyectos, especialmente si se mejora el lado humano de su implementación.

Hiatt (2006) considera que cuando se hace énfasis en los procesos en lugar de la gente, no se toma en cuenta al cambio como un complejo y sistémico fenómeno que involucra a una multiplicidad de variables interdependientes, y no se destaca la diferencia que existe entre los cambios individuales y organizacionales.

Según Tréhorel (2007), la gestión de proyectos y la gestión del cambio se han convertido en el nuevo reto de las organizaciones; ellas quieren cambios más exitosos y buscan en la gestión de proyectos la manera de lograr un mejor desempeño. Parker, Charlton, Ribeiro, \& Pathak (2013) consideran que es muy importante para las organizaciones la utilización de iniciativas basadas en proyectos como palancas del cambio organizacional para asegurar su éxito. Kerzner (2013) destaca el importante impacto que tienen el cambio organizacional y la cultura en la iniciación, procesamiento e implementación de proyectos.

Las organizaciones en su mayoría no tienen como base de su estructura a los proyectos; muchas adoptan un enfoque típicamente funcional, con áreas o departamentos claramente diferenciados. Sin embargo, pocas privilegian la consecución de proyectos, con estructuras matriciales en donde cada una de las áreas se convierte en un apoyo; su enfoque no es el de funciones diferenciadas y divorciadas entre sí; por el contrario, todas contribuyen a que el proyecto logre su propósito. Para Abrantes \& Figueiredo (2013), este segundo tipo de organizaciones basadas en proyectos deben estar preparadas para ambientes de innovación, en donde el cambio es una necesidad permanente. La flexibilidad y la capacidad de adaptación, tanto de los procesos como de sus estructuras, las preparan para los cambios, les permite tomar ventaja de las oportunidades, evolucionar y las vuelve capaces de aprovechar los cambios, lo cual es crítico para su éxito.

Para Klein, Biesenthal, \& Dehlin (2015), la gestión de proyectos es compleja y, por lo tanto, es un terreno fértil para la creatividad, espontaneidad y aplicación intuitiva de teorías particulares para satisfacer los objetivos establecidos en un ambiente de constante cambio. Para estos autores, esta forma de trabajo tiene un componente de improvisación, ya que implica un acercamiento pragmático a la aplicación de las teorías existentes en nuevas maneras de alcanzar el éxito del proyecto. Cunha $\&$ Cunha (2006) consideran que la capacidad de recuperación, llamada resiliencia, se convierte en la respuesta al problema de la complejidad y, conjuntamente con la improvisación, son los medios para adaptar la estructura y los procesos existentes a fin de entregar un proyecto exitoso.

Rosenbloom \& Markus (2010) sostienen que el cambio es un camino, que consiste en cómo los individuos transitan y responden al mismo (Figura 2).

$\mathrm{Al}$ inicio el cambio es inconsciente, los involucrados no tienen conocimiento ni conciencia que está llegando. Una vez que los involucrados se dan cuenta que algo ocurre o está por ocurrir, el cambio se vuelve consciente. Cuando los involucrados dejan de ver al cambio como algo meramente conceptual y pueden procesar lo que este realmente significa para ellos, tanto personal como profesionalmente, entonces se ha llegado al entendimiento del cambio. Al momento en que los involucrados comienzan a tomar acciones de respuesta con respecto del cambio, entonces se puede decir que lo han aceptado. Finalmente, si los involucrados se comprometen con el cambio, lo promueven, lo comunican e inspiran a que otros lo acepten, entonces se podrá decir que se han apropiado del cambio.

Gerdin et al. (2010) proponen las siguientes recomendaciones para gestionar el cambio en las organizaciones hacia un enfoque basado en proyectos:

- Identificar y contratar a las personas claves. 
FIgURA 2.

LA CURVA DEL CAMBIO

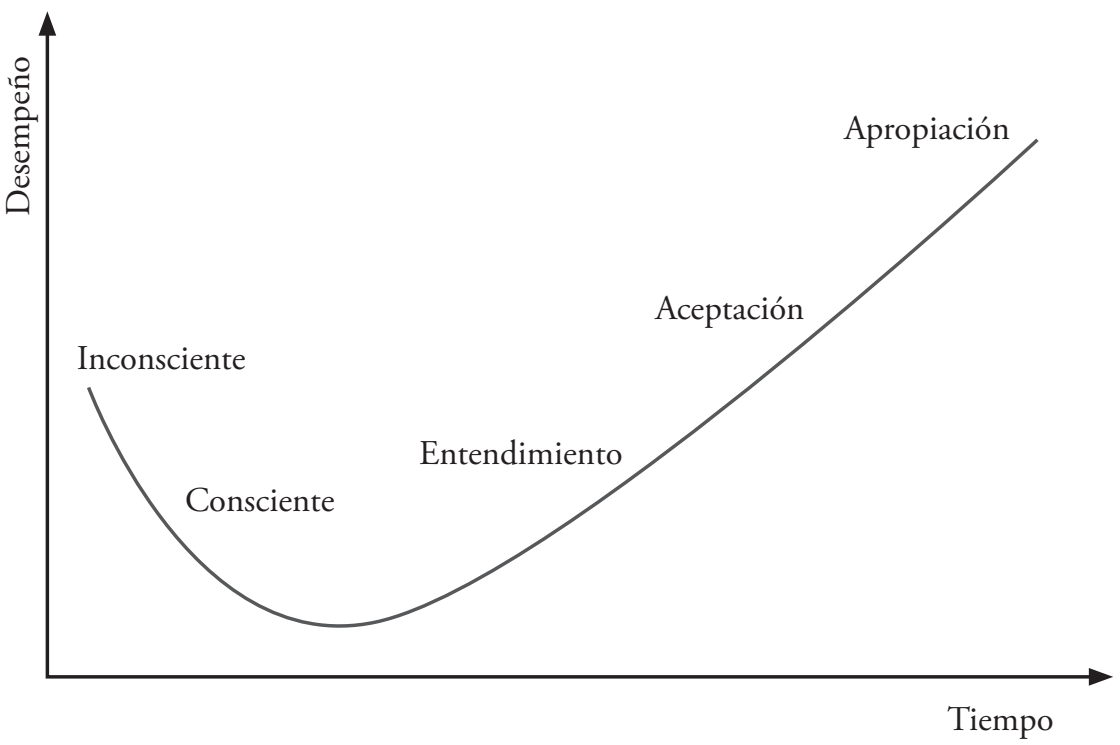

Fuente: Rosenbloom \& Markus (2010)

- Encontrar las verdaderas fuentes de motivación.

- Evitar la implementación de cambios dirigida estrictamente desde arriba hacia abajo.

- Diseñar un plan de implementación conjuntamente entre los líderes de los proyectos y su equipo.

- Priorizar las iniciativas de cambio.

- Crear las condiciones necesarias para permitir el intercambio de experiencias.

- Adaptar la visión y los medios de comunicación con respecto de las diferencias verticales y horizontales de este tipo de organizaciones.

- Establecer metas claras de corto plazo y recompensas que sean alcanzables.

\section{Aprendizaje organizacional de los proyectos}

Aramburu Goya (2000) considera que existen dos perspectivas desde las que se aborda el estudio del aprendizaje en las organizaciones: el cambio y el conocimiento. La perspectiva del cambio considera que una organización aprende en la medida que esta es capaz de adaptarse a su entorno "visión adaptativa» o promover su propia transformación "visión proactiva». La perspectiva del conocimiento propone que el aprendizaje está ligado a los procesos a través de los cuales la organización crea o construye conocimientos y amplía la base de estos.

Chronéer \& Backlund (2015) sostienen que el aprendizaje en las organizaciones basadas en proyectos es muy importante, pero difícil de ponerlo en práctica. Estos autores creen que es necesario tener un "pensamiento de procesos" que soporte el aprendizaje y la retroalimentación de la experiencia, además del típico "pensamiento de proyectos», que está relacionado con sus fases: inicio, planeación, ejecución, monitoreo y control, y cierre. Esta mirada por procesos debería soportar el aprendizaje, no solo al cierre del proyecto, sino también entre sus fases. De esta manera la naturaleza temporal de los proyectos se complementaría con el carácter continuo de los procesos con el propósito que el conocimiento permanezca dentro de la organización.

Para Anderson \& Robbins (2001) las organizaciones se aproximan al problema de la gestión del conocimiento desde dos tipos de iniciativas: captura del conocimiento tácito y construcción de una infraestructura de gestión del conocimiento. La primera, busca promover la transmisión del conocimiento de las experiencias de los indivi- 
duos; mientras que la segunda, trata de mejorar los procesos para la transmisión del conocimiento explícito. Estos autores proponen que ambas iniciativas deben complementarse para que se puedan gestionar de manera eficiente los procesos que permitan trasmitir los conocimientos explícitos y tácitos, lo cual beneficia a la gestión de proyectos.

Hanisch et al. (2008) identifican los siguientes retos de la gestión del conocimiento en las organizaciones basadas en proyectos:

- Una vez que el proyecto termina el equipo del proyecto se dispersa, por lo que el aprendizaje y la construcción de equipos de un proyecto a otro se dificulta.

- Los proyectos están relacionados con una fuerza de trabajo cambiante, lo que desemboca en que muchos de los conocimientos adquiridos queden en el olvido, ante la falta de rutinas que los afiancen y conviertan en sostenibles a las experiencias aprendidas.

- La gente en los proyectos tiene que adaptarse rápidamente a nuevas condiciones generales y contenidos de trabajo.

- Las actividades de gestión del conocimiento suelen tener una perspectiva de largo plazo, mientras que la gestión de proyectos tiene una de corto plazo, dada su naturaleza temporal. Es necesario integrar los objetivos de conocimiento a los objetivos del proyecto y especificar las responsabilidades y procesos de la gestión del conocimiento entre la organización primaria y la organización del proyecto.

Johansson, Moehler, \& Vahidi (2013) proponen un esquema que resume los elementos relacionados con el aprendizaje en los proyectos (Figura 3).

\section{Figura 3. Elementos de LA gestión de CONOCimiento del PROYeCto}

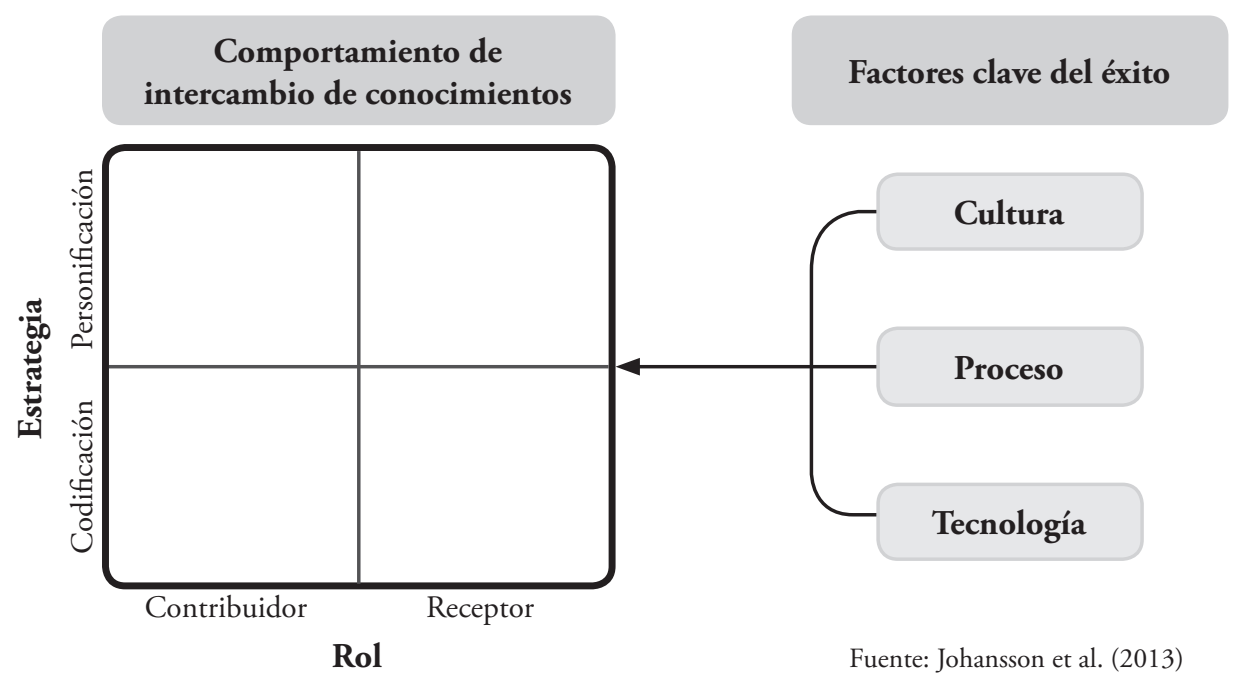

En el esquema se puede apreciar que las personas dentro del proyecto actúan como contribuidores cuando comparten su conocimiento, pero cuando utilizan el conocimiento que les ha sido compartido, estos actúan como receptores.

Para compartir el conocimiento, la estrategia de codificación utiliza documentos, en los que reposa el conocimiento explícito previamente co- dificado. La estrategia de personalización transfiere el conocimiento mediante la interacción personal, lo cual permite compartir el conocimiento tácito. Ambas estrategias se complementan, la codificación permite compartir grandes cantidades de conocimiento de manera eficiente, mientras que la personalización permite que el conocimiento sea adaptado a las necesidades de cada receptor. 
En relación con los factores claves de éxito que influencian en el uso de las estrategias de intercambio de conocimiento entre el contribuidor y el receptor, destaca la cultura como el factor más importante. Por su parte, los procesos se refieren a las guías que facilitan la generación, almacenamiento, diseminación y recuperación del conocimiento del proyecto. Finalmente, la tecnología es la herramienta que soporta la compartición del conocimiento, pero a pesar de ser útil y necesaria, no es un factor suficiente para tener calidad en la gestión del conocimiento del proyecto. La armonía entre los tres factores es lo que asegura el éxito de la gestión de conocimiento del proyecto.

Fernández Gatti (2007) propone un proceso de aprendizaje trasformador, en donde el diálogo entre los agentes es crucial. En una organización basada en proyectos, los agentes son todos sus involucrados, entre los que se debe propiciar un diálogo generativo que permita alinear y consensuar sus acciones a través del interaprendizaje. Para que esto sea posible, es necesario que se favorezca el proceso de comunicación dentro de la organización basada en proyectos. El PMI (2007) considera a la comunicación como una de las competencias que el director de proyectos debe desarrollar, entendiéndose a esta como la capacidad de asegurar los intercambios efectivos, apropiados y relevantes de información con los involucrados usando métodos apropiados. Según Matteucci (2001), las deficiencias en la comunicación son la tercera causa del fracaso de los proyectos. Davis-Muffet (1997) considera que las tácticas comunicacionales deben enfocarse en una o más audiencias de involucrados objetivos, enviándoles un mensaje claro y comunicar de una forma que estos puedan entenderlo y apreciarlo. Para tal efecto, se deben desarrollar mensajes claves que el proyecto reconozca como información crítica. La comunicación debe ser directa, no demasiado técnica, basada en la realidad y corta. Puede ser necesario desarrollar subgrupos de mensajes claves específicamente diseñados para ciertos involucrados, pero que deben estar claramente alineados al paraguas general de los mensajes claves del proyecto. Para operacionalizar estas tácticas, es necesario que se identifique dentro de la organización a profesionales con la experiencia y el conocimiento para desarrollar las estrategias sobre cómo comunicarse con los distintos grupos de involucrados.

Morris (2002) considera que la complejidad de la gestión del conocimiento plantea los siguientes retos para la gestión de proyectos contemporánea, en cuanto a su desarrollo e investigación:

- La búsqueda actual de las mejores prácticas de proyectos que permitan identificar criterios de desempeño para proyectos de clase mundial, es una quimera. Probablemente sea más adecuado hablar de mejores proyectos dentro de su propia clase y considerar a la excelencia como una función de aprendizaje dentro de los procesos de gestión, que permitan al proyecto operar y descubrir sus necesidades de una manera más adecuada.

- La idea de una mejora progresiva del desempeño a través de niveles de madurez de las capacidades, aunque podría ser útil en los niveles más bajos de desarrollo, se hace más compleja en los niveles más altos de capacidad. Es más realista el modelo de espiral propuesto por Nonaka (1994), debido a que la gestión del aprendizaje está relacionada con la optimización del rendimiento en un contexto específico, para lo cual se requiere reflexión e iteración, basada en una apreciación justa de los fundamentos.

- Cuando el intercambio de conocimientos es económicamente atractivo, las condiciones a través de todo el proyecto, incluyendo la cadena de suministro, deben estar alineadas para apoyar este intercambio. Es más probable que una empresa acumule el conocimiento que es altamente específico e importante para su competitividad y que, a su vez, comparta el conocimiento que se encuentra difuso.

- Es difícil relacionar el aprendizaje del proyecto con el aprendizaje organizacional a nivel estratégico de la empresa. Las empresas encuentran más difícil el aprendizaje de carácter estratégico de los proyectos, mientras que para los proyectos es más fácil aplicar el conocimiento estratégico de las empresas. 


\section{Conclusiones}

La gestión por proyectos es manera de administrar, que poco a poco se va adentrando en las organizaciones, como una respuesta a la necesidad de cumplir con sus objetivos organizacionales de una manera más eficaz y eficiente, frente a los retos que plantea el contexto actual. Sin embargo, para que esta se implemente de manera adecuada, es necesario que las organizaciones atraviesen por un proceso de cambio en el cual aprendan a desarrollar una cultura basada en proyectos.

La cultura basada en proyectos tiene claramente definidos sus propios procesos, así como sus valores específicos y sus presunciones intrínsecas, los mismos que le han permitido resolver los problemas que a un proyecto se le presentan a lo largo de su ciclo de vida. Para que una organización adopte este tipo de cultura, esta debe favorecer un ambiente flexible y dinámico, en el cual exista un equilibrio por la creación de conocimiento enfocada a una visión compartida, así como la creatividad que nace desde la motivación personal de cada uno de los colaboradores. Es importante que todos estén involucrados y comprendan claramente el valor, el alcance y los riesgos que esta cultura implica.

Para que una organización adopte una cultura basada en proyectos es necesario que el proceso de cambio sea planificado y permanentemente monitoreado, lo cual permita resolver los inconvenientes que puedan surgir a lo largo de su implementación y obtener los beneficios esperados. Este proceso puede iniciar de manera inconsciente, pero para gestionarlo de manera eficaz, es necesario establecer las acciones para que lo vuelvan consciente, permitiendo su real entendimiento, aceptación y apropiación. Solo cuando la cultura basada en proyectos se convierta en el paradigma organizacional para la toma de decisiones y la solución de problemas, entonces se podrá decir que esta ha sido plenamente incorporada al quehacer cotidiano. Los directores de proyecto tienen la responsabilidad de llevar este tipo de patrones desde sus proyectos hasta la organización matriz, de tal manera que lleguen a formar parte de la cultura organizacional y no se queden solamente como parte de proyectos específicos aislados.

El tránsito hacia una cultura basada en proyectos implica un cambio en donde la capacidad de aprendizaje de la organización es fundamental. De esta manera, se procurará desde un inicio la búsqueda de adeptos y su apoyo, para luego atravesar por un camino de transformación debidamente planificado, socializado e implementado, que pueda ser monitoreado y que permita que los efectos de los cambios introducidos permanezcan en la organización formando parte de su cultura.

Dada la tendencia de las organizaciones por desarrollar una gestión total de proyectos, se identifica la necesidad de analizar de manera detallada las razones y resultados de aquellas que se han decidido por esta modalidad, para observar el tratamiento que han dado a sus aspectos culturales, sus modos de aprendizaje y sus procesos de cambio, a lo largo de la implementación de esta cultura. Esto coadyuvará a que las organizaciones que pretendan adoptar esta modalidad de gestión en nuestras economías, desarrollen estrategias mejor dirigidas que ayuden a minimizar los riesgos que implica el cambio hacia la cultura basada en proyectos y que puedan aprovecharse de los beneficios que esta puede brindar. 


\section{Referencias bibliográficas}

- Abrantes, R., \& Figueiredo, J. (2013). Preparing Project based Organizations for Change. Procedia Technology, 9, 757-766. http://doi. org/10.1016/j.protcy.2013.12.084

- Ajmal, M. M., \& Koskinen, K. U. (2009). Knowledge Transfer in Project - Based Organizations: An Organizational culture Perspective. Project Management Journal, 39 (March), 1-11. http://doi.org/10.1002/pmj

- Anderson, A., \& Robbins, G. (2001). Building a Knowledge Culture. In PMI Annual Seminars \& Symposium (pp. 1-6). Nashville.

- Aramburu Goya, N. (2000). Un Estudio del Aprendizaje Organizativo desde la Perspectiva del Cambio: Implicaciones Estratégicas y Organizativas. Universidad de Deusto.

- Chronéer, D., \& Backlund, F. (2015). A Holistic View on Learning in Project-Based Organizations. Project Management Journal, 46 (3), 61-74. http://doi.org/10.1002/pmj.21503

- Cunha, M. P. e, \& Cunha, J. V. da. (2006). Towards a complexity theory of strategy. $M a$ nagement Decision, 44 (7), 839-850. http:// doi.org/10.1108/00251740610680550

- Davis-Muffet, P. (1997). Communications as a strategic PM function. Strategy Leadership, $25,44$.

- Dinsmore, P. C. (1997). Toward a Corporate Project Management Culture. In PMI 28th Annual Seminars \& Symposium (pp. 1-7). Chicago.

- Domingo Ajenjo, A. (2000). Dirección y Gestión de Proyectos. (Alfaomega, Ed.) (1st ed.). México.

- Fernández Gatti, M. A. (2007). Empresas de Rosario: un modelo de cambio basado en el aprendizaje. Universidad del Rosario.
- Gerdin, C., Björkander, M., Henriksson, M., \& Nilsby, P. (2010). Change Management In Project Based Organizations A Case Study Of A Construction Company. PMI.

- Haimes, G. (2001). Project culture. In PMI Annual Seminars \& Symposium (pp. 1-8). Nashville.

- Hanisch, B., Lindner, F., Müller, A., \& Wald, A. (2008). Project knowledge management. Oestrich-Winkel.

- Happy, R., \& Meyer, C. (2001). Establishing an Effective PM Culture. In PMI Annual Seminars \& Symposium (pp. 1-7). Nashville.

- Hiatt, J. M. (2006). ADKAR: A Model For Change In Business, Government And Our Community: How To Implement Successful Change In Our Personal.

- Hobday, M. (2000). The project-based organisation: an ideal form for managing complex products and systems? Research Policy, 29 (78), 871-893. http://doi.org/10.1016/S00487333(00)00110-4

- ISO 10006. (2003). ISO 10006:2003 Sistemas de gestión de la calidad - Directrices para la gestión de la calidad en los proyectos.

- Johansson, T., Moehler, R. C., \& Vahidi, R. (2013). Knowledge Sharing Strategies for Project Knowledge Management in the Automotive Sector. Procedia - Social and Behavioral Sciences, 74, 295-304. http://doi.org/10.1016/j.sbspro. 2013.03.018

- Kerzner, H. R. (2013). Project management: a systems approach to planning, scheduling, and controlling. John Wiley \& Sons.

- Klein, L., Biesenthal, C., \& Dehlin, E. (2015). Improvisation in project management: A praxeology. International Journal of Project 
Management, 33 (2), 267-277. http://doi. org/10.1016/j.ijproman.2014.01.011

- Levasseur, R. E. (2010). People Skills: Ensuring Project Success- A Change Management Perspective. Interfaces, 40 (2), 159-162. http:// doi.org/10.1287/inte.1090.0473

- Lindkvist, L. (2004). Governing Project-based Firms: Promoting Market-like Processes within Hierarchies. Journal of Management \& Governance, 8 (1), 3-25. http://doi.org/10.1023/ B:MAGO.0000015392.75507.ad

- Matteucci, N. (2001). Virtual project Managemnt. Project World.

- Moran, J. W., \& Brightman, B. K. (2001). Leading organizational change. Career Development International, 6 (2), 111-119. http:// doi.org/10.1108/13620430110383438

- Morris, P. W. G. (2002). Managing PM Knowledge for Organizational Effectiveness.

- Nonaka,I. (1994). A dynamic theory of organizational knowledge creation. Organization Science, 5 (1), 14-37. Retrieved from http://papers. ssrn.com/sol3/papers.cfm?abstract_id=889992

- OGC. (2009). Glosary of Terms, PRINCE 2:2009.
- Parker, D., Charlton, J., Ribeiro, A., \& Pathak, R. D. (2013). Integration of project-based management and change management. International Journal of Productivity and Performance Management, 62 (5), 534-544. http://doi. org/10.1108/IJPPM-10-2012-0108

- PMI. (2007). Project Manager Competency Development (PMCD) Framework (2nd ed.). Pennsylvania: Project Management Institute.

- PMI. (2013). Fundamentos para la dirección de proyectos (Guía del PMBOK) (5th ed.). Pennsylvania.

- Rosenbloom, S., \& Markus, M. J. (2010). When Project and Organizational Culture Clash. In PMI Global Congress Proceedings. Washington DC.

- Schacht, N. R. (1997, September). Project Management Culture. PM Network, pp. 1-6.

- Schein, E. H. (2009). The Corporate Culture Survival Guide. John Wiley \& Sons.

- Schneider, W. E. (1994). The reengineering alternative: A plan for making your current culture work. Richard D Irwin.

- Tréhorel, Y. (2007). Mettez du changement dans vos projets! Afnor. 